\title{
0 campo do fluxo de vapor dágua atmosférico sobre a região Amazônica
}

\author{
José Marques ( ${ }^{*}$ ) \\ Jesus Marden dos Santos (**) \\ Eneas Salati $\left({ }^{* *^{*}}\right)$
}

\begin{abstract}
Resumo
Utilizando dados de ar superior obtidos em 9348 radiossondagens na regiāo Amazônica e adjacências, durante o período $1972 / 1975$, foram estimadas as componentes zonal e meridional do fluxo de vapor $\vec{Q}$, integrado entre os niveis de 1000 (superfície) e 500 milibares (5560 metros de altitude, aproximadamente). Ficou evidenciado o comportamento predominantemente zonal do fluxo na região, estimando-se em 2895 e $2203 \mathrm{~g}$ da vapor $/ \mathrm{cm}$.s os valores médios da componente zonal em Belém e Manaus, respectivamente.
\end{abstract}

\section{INTRODUÇÃo}

A precipitação média da região Amazônica é em torno dos $2400 \mathrm{~mm} /$ ano, constituindo um dos mais altos índices pluviométricos do giobo. A questão fundamental é saber qual a real contribuição da floresta para esta precipitação. A hidrologia clássica, utilizando métodos que perrnitem o estudo do balanço de água de uma bacia hidrológica, não fornece informações quanto à origem do vapor dágua atmosférico que realmente controla o ciclo hidrológico.

Os estudios da circulação geral da atmosfera conduziram a uma nova metodologia de pesquisas hidrológicas baseada na análise dos campos das diversas grandezas que caracterizam o estado da atmosfera e a dinâmica da água na fase de vapor.

Com o aumento do número de observaçōes do ar superior verificado no mundo, tem sido possível solucionar muitos problemas associados ao transporte de vapor dágua atmosférico e suas conseqüências no balanço da água em uma região. Esta metodologia foi aplicada à bacia Amazônica e algumas informações básicas puderam ser obtidas quanto à dinâmica do vapor dágua na região. Os dados obtidos de uma análise de 9348 radiossondagens em 4 anos, serão publicados em 4 artigos. Este é o primeiro, versando sobre o transporte de vapor dágua; o segundo sobre o armazenamento aéreo do vapor dágua; o terceiro sobre a divergência do fluxo de vapor dágua e as chuvas na região e, finalmente, no quarto far-se-á uma estimativa da evapotranspiração real na bacia.

\section{REVISÃO DA LITERATURA}

Estudos recentes mostram que o transporte aéreo de vapor constitui o mais importante suprimento de água para a seqüência do ciclo hidrológico. Vários estudos sobre o transporte aéreo de vapor dágua foram feitos, dentre outros, por Benton \& Estoque (1954) e Rasmusson $(1966,1967)$ para os Estados Unidos; para o mar Báltico por Palmén \& Söderman (1966); para a Austrália por Hutchings (1961) e para a bacia Amazônica por Molion (1975), Marques (1976) e Marques et al. (1977) .

Benton et al. (1950) evidenciaram o papel do vapor dágua atmosférico no ciclo hidrológico e esclareceram as relações entre o ciclo e a massa de ar que se deslocam sobre a bacia do rio Mississipi. A partir de dados aerológicos os autores determinaram que não mais que $10 \%$ da precipitação total é oriunda de vapor dágua continental e que a maior parte da chuva caída originou-se da massa de ar oceânica. Segundo eles, não fơi necessária urra alta correlação entre os valores mensais da transferência do vapor dágua e da precipitação; mas a precipitação foi mais associada com a convergência do campo do vapor dágua do que com a sua transferência, idéia esta con-

(*) - UFRJ - I. Geociências, Departamento de Meteorologia

( *) - ESALQ/USP - Departamento de Física e Meteorologia

(**) - Instituto Nacional de Pesquisas da Amazônia, Manaus. 
firmada em trabalhos de Spar (1953), Benton \& Estoque (1954), Peixoto (1959), Bettencourt \& Faria (1970) e Barnes (1964).

\section{MATERIAL E MÉTODOS}

Os dados básicos de altitude utilizados neste estudo foram obtidos a partir de informaçōes aerológicas das seguintes estaçōes. cuja localização acha-se na figura 1 :

\begin{tabular}{|c|c|c|c|c|}
\hline Estação & País & $\begin{array}{c}\text { Altitude } \\
\mathrm{m}\end{array}$ & Latitude & Logintude \\
\hline Belém & Brasil & 40 & $1^{\circ} 28^{\prime} \mathrm{S}$ & $48^{\circ} 27^{\prime} \mathrm{W}$ \\
\hline Brasília & Brasil & 1061 & $15 \circ 50^{\prime} \mathrm{S}$ & $48 \circ 00^{\prime} \mathrm{W}$ \\
\hline Carolina & Brasil & 193 & $7 \circ^{\circ} 0^{\prime} \mathrm{S}$ & $47 \circ 28^{r} \mathrm{~W}$ \\
\hline Manaus & Brasil & 13 & 3०09'S & $59 \circ 59^{\prime} \mathrm{W}$ \\
\hline Vilhena & Brasil & 582 & $12 \circ 44^{\prime} \mathrm{S}$ & $60 \circ 08^{\prime} \mathrm{W}$ \\
\hline Bogotá & Colômbia & 2541 & $4 \circ 42 \mathrm{~N}$ & $74^{\circ} 09^{\prime} \mathrm{W}$ \\
\hline Lima & Peru & 13 & $12^{\circ} 01^{\prime} \mathrm{S}$ & $77 \circ 08^{\prime} W$ \\
\hline Iquitos & Peru & 126 & $3^{\circ} 45^{\prime S}$ & $73 \circ 15^{\prime} \mathrm{W}$ \\
\hline Maracay & Venezuela & 442 & $10^{\circ} 20^{\prime} \mathrm{N}$ & $66^{\circ} 45^{\prime} \mathrm{W}$ \\
\hline S. Antonio & Veneztiela & 404 & $8^{\circ} 00^{\prime} \mathrm{N}$ & $72 \circ 30^{\prime} \mathrm{W}$ \\
\hline S. Elena & Venezuela & 907 & $4 \circ 35^{\prime} \mathrm{N}$ & $61000^{\prime} \mathrm{W}$ \\
\hline
\end{tabular}

O fluxo de vapor dágua $\vec{Q}$ entre dois niveis isobáricos atmosféricos $P_{1}$ e $P_{2}$ é dado pela seguinte equação :

$$
\vec{Q}=\frac{1}{g} \int_{P_{1}}^{P_{2}} \bar{q} \overrightarrow{V d P}
$$

onde :

$$
\begin{aligned}
& \vec{v}=\text { componente horizontal do } \\
& \text { vento } \mathrm{em} \mathrm{m} / \mathrm{s} \text {; } \\
& \mathrm{g}=\text { aceleração da gravidade em } \\
& \mathrm{m} / \mathrm{s}^{2} \text {; } \\
& \bar{q}=\text { umidade especifica média } \\
& \text { entre os niveis } P_{1} \text { e } P_{2} \text {, em }
\end{aligned}
$$

Observações diárias do vento, da temperatura e da umidade relativa disponíveis para até 8 níveis isobáricos estendendo-se desde a superfície até os $500 \mathrm{mb}(\simeq 5.500 \mathrm{~m})$, foram utilizados para computar a transferência horizontal de vapor dágua na área coberta pelas estações aerológicas acima relacionadas.

Computou-se, os fluxos parciais $\vec{Q}$, em cada subcamada de $50 \mathrm{mb}$ de espessura com os dados obtidos em cada estação aerológica isola damente; os valores $\vec{Q}$ ' integrados na vertical. foram utilizados para estimar o fluxo total de vapor sobre cada estação.

Foram utilizados os dados referentes ao período $1972 / 1975$, num total de 9348 radios. sondagens .

$O$ vento horizontal $\vec{V}$ foi decomposto nas suas componentes zonal (u) e meridional (v) ou seja, $\vec{v}=\overrightarrow{u i}+\overrightarrow{v j}$ obtendo-se assim, a partir da equação 1 , as correspondentes componentes zonal $\mathrm{Q}_{\lambda}$ (positiva no sentido oeste-leste) e meridional $Q_{\phi}$ (positiva no sentido sulnurte) do fluxo:

$$
\begin{aligned}
& \mathrm{Q}_{\lambda}=\frac{1}{\mathrm{~g}} \int \overline{\mathrm{qu}} \mathrm{dp} \ldots \ldots \ldots \ldots \\
& \mathrm{Q}_{\dot{\phi}}=\frac{1}{\mathrm{~g}} \int \overline{\mathrm{qv}} \mathrm{dp} \ldots \ldots \ldots \ldots
\end{aligned}
$$

No presente trabalho, $P_{1}=1000 \mathrm{mb}$ e $P_{2}=$ $500 \mathrm{mb}$. Comio a variação vertical da umidade específica (q) não é linear entre esses dois níveis, utilizou-se os valores obtidos para as subcamadas $1000 / 950,950 / 900,900 / 850,850 /$ $800,800 / 700,700 / 600$ e $600 / 500 \mathrm{mb}$.

Os valores das componentes do fluxo foram lançados em mapas e traçados os respectivos campos dos fluxos zonal e meridional. Por leitura direta em cada vértice de uma malha de $5^{\circ}$ de lado foram obtidos os valores das componentes do fluxo e obteve-se o valor do fluxo total de vapor $\mathrm{Q}=\sqrt{\mathrm{Q}_{\lambda}^{2}+\mathrm{Q}_{\phi}^{2}}$ em cada ponto.

\section{RESUltados OBTidos E Discussão}

Acima do nivel dos $500 \mathrm{mb}$ as curvas mostraram uma tendência para serem nulos os valores da umidade específica $q$, razão pela qual os estudos se restringiram somente até este nivel. 


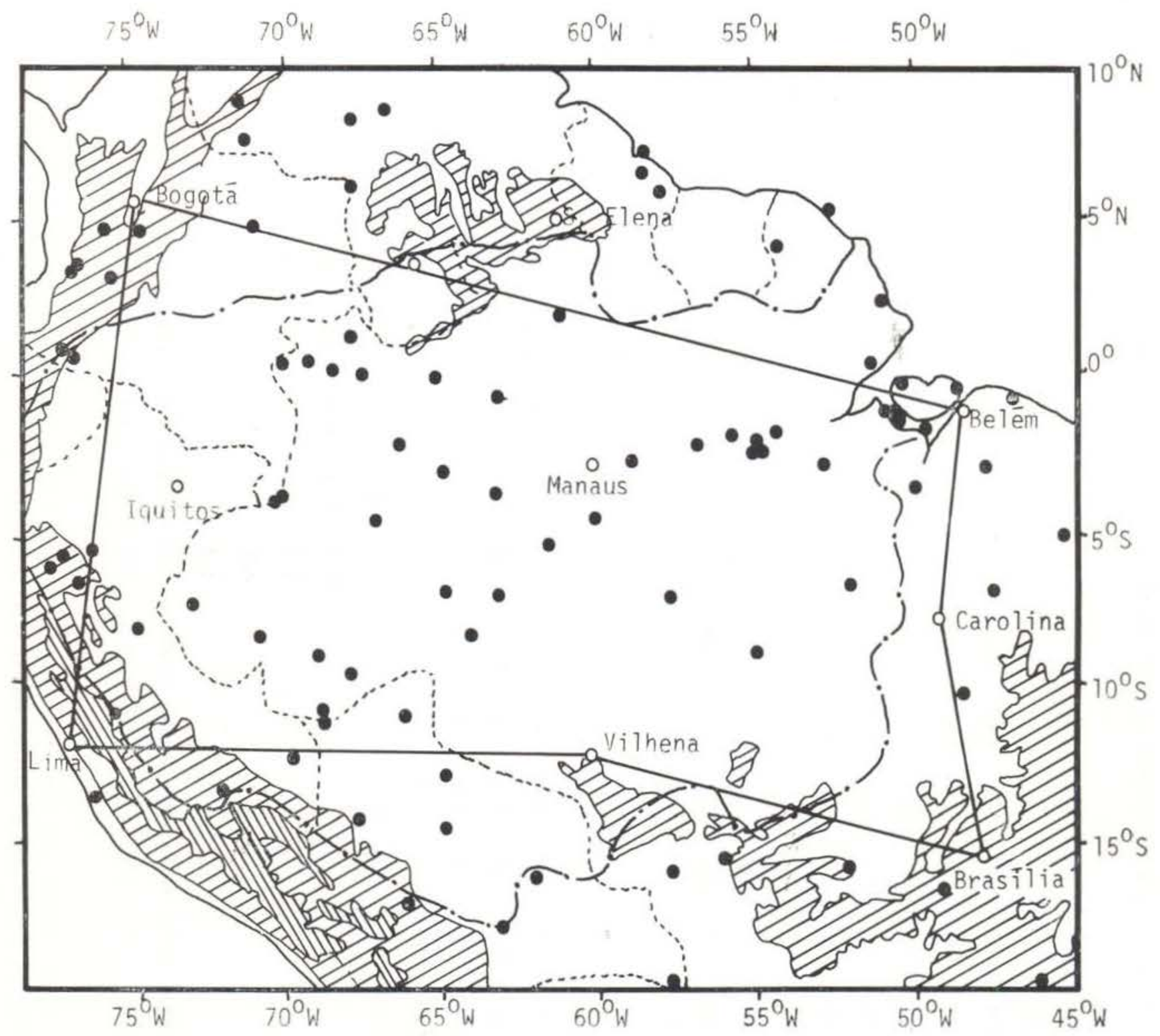

27 Altitudes entre 500 e 1000 metros

MIIIV Altitudes acima de 4000 metros

- Estaçoes pluviométricas

- Estacoes de radiossondagens

- Limites da bacia Amazônica

Fig. 1 - Hede de estaçōes pluviométricas e de radiossondagens utilizadas.

o campo do... 
IABELA 1 - Valores mensais e médios das componentes zonal $Q_{\lambda}$ e meridional $Q_{\phi}$, em gramas de vapor/cm.s, do fluxo de vapor no periodo $1972 / 1975$.

\begin{tabular}{|c|c|c|c|c|c|c|c|c|c|c|c|c|c|c|c|c|}
\hline Estação & Componente & Jan & Fev & Mar & Abr & Mai & Jun & Jul & Ago & Set & Out & Nov & Dez & Média & $\begin{array}{c}\mathrm{N} . \circ \text { de } \\
\text { sondagens }\end{array}$ & OBS. \\
\hline \multirow[t]{2}{*}{ Belém } & $\mathrm{Q}_{\lambda}$ & -3024 & -2914 & -2744 & -2655 & -2847 & -3185 & -2977 & -2979 & -3019 & -2731 & -2835 & -2725 & -2895 & 1201 & \\
\hline & $\mathrm{Q}_{\phi}$ & 283 & 321 & 48 & 390 & 844 & 1126 & 1447 & 886 & 662 & 19 & 123 & 123 & 523 & & \\
\hline \multirow[t]{2}{*}{ Bogotá } & $Q_{\lambda}$ & -358 & -265 & -302 & -351 & -647 & -896 & -859 & -789 & -553 & -379 & -409 & -267 & -506 & 1337 & \\
\hline & $\mathrm{Q}_{\phi}$ & 97 & 103 & 65 & 125 & 191 & 234 & 255 & 219 & 205 & 72 & 44 & 48 & 138 & & \\
\hline \multirow[t]{2}{*}{ Brasilia } & $Q_{\lambda}$ & -560 & -841 & -141 & -474 & -505 & -443 & -468 & -405 & .719 & 29 & 256 & -254 & -371 & 2314 & \\
\hline & $\mathrm{Q}_{\Phi}$ & -585 & -444 & 655 & -200 & -76 & -87 & -127 & 23 & 19 & -330 & -549 & -696 & -309 & & \\
\hline \multirow[t]{2}{*}{ Carolina } & $Q_{\lambda}$ & -1105 & -531 & .825 & .1310 & -1825 & -1627 & 856 & -344 & -1190 & -905 & -1107 & -413 & -1003 & 977 & \\
\hline & $\mathrm{O}_{\phi}$ & -308 & -104 & -226 & 64 & 696 & 646 & 165 & 52 & 44 & -197 & -228 & -231 & 15 & & \\
\hline \multirow[t]{2}{*}{ Iquitos } & $\alpha_{\lambda}$ & & & & & & & & & -1411 & -826 & -979 & -818 & -1008 & 54 & (3) \\
\hline & $\mathrm{Q}_{\phi}$ & & & & & & & & & -235 & -1265 & -1243 & -1058 & -950 & & (3) \\
\hline \multirow[t]{2}{*}{ LIma } & $\alpha_{\lambda}$ & -174 & -99 & .44 & -118 & -163 & -124 & -201 & -148 & -142 & -163 & -204 & -226 & -150 & 1333 & \\
\hline & $\mathrm{Q}_{\phi}$ & 183 & 166 & 31 & 70 & 103 & 139 & 175 & 170 & 89 & 203 & 216 & 326 & 156 & & \\
\hline \multirow[t]{2}{*}{ Manaus } & $Q_{\lambda}$ & -2229 & -2330 & -1869 & -1852 & -2354 & -2743 & -2670 & -2346 & -2345 & -1879 & -1791 & -2029 & -2203 & 1081 & \\
\hline & $\mathrm{Q}_{\Phi}$ & -505 & -504 & -460 & -101 & 114 & 499 & 717 & -304 & 274 & -209 & 68 & -353 & -64 & & \\
\hline \multirow[t]{2}{*}{ Maracay } & $\mathrm{O}_{\lambda}$ & -561 & -310 & -245 & .220 & -1019 & -1937 & & -1495 & -1272 & -958 & -874 & -712 & -873 & 487 & (5) \\
\hline & $\mathrm{Q}_{\phi}$ & 91 & 19 & 73 & 194 & 774 & 1105 & & 640 & 879 & 490 & 181 & 101 & 413 & & (5) \\
\hline \multirow[t]{2}{*}{ S. Antonio } & $O_{\lambda}$ & & & & & & & & & -1238 & -1517 & -747 & -34 & -884 & 74 & (1) \\
\hline & $\mathrm{Q}_{\phi}$ & & & & & & & & & 887 & 710 & 473 & -215 & 464 & & (1) \\
\hline \multirow[t]{2}{*}{ S. Elena } & $\mathrm{Q}_{\lambda}$ & & & & & & & & & -743 & & & & & 7 & (2) \\
\hline & $\mathrm{Q}_{\phi}$ & & & & & & & & & 234 & & & & & & (2) \\
\hline \multirow[t]{3}{*}{ Vilhena } & $\mathrm{O}_{\lambda}$ & 108 & 449 & 31 & 116 & -535 & -750 & -356 & -573 & -643 & -278 & 250 & 633 & -129 & 483 & (4) \\
\hline & $\mathrm{O}_{\phi}$ & -824 & .771 & -570 & -37 & 385 & -703 & -523 & -635 & -716 & -625 & -848 & -182 & -568 & & (4) \\
\hline & & & & & & & & & & & & & & Total & 9348 & \\
\hline
\end{tabular}

(1) somente 1973 e 1974; (2) somente 1974; (3) somente 1975; (4) somente 1972 e 1973; (5) somente 1972, 19731974. 


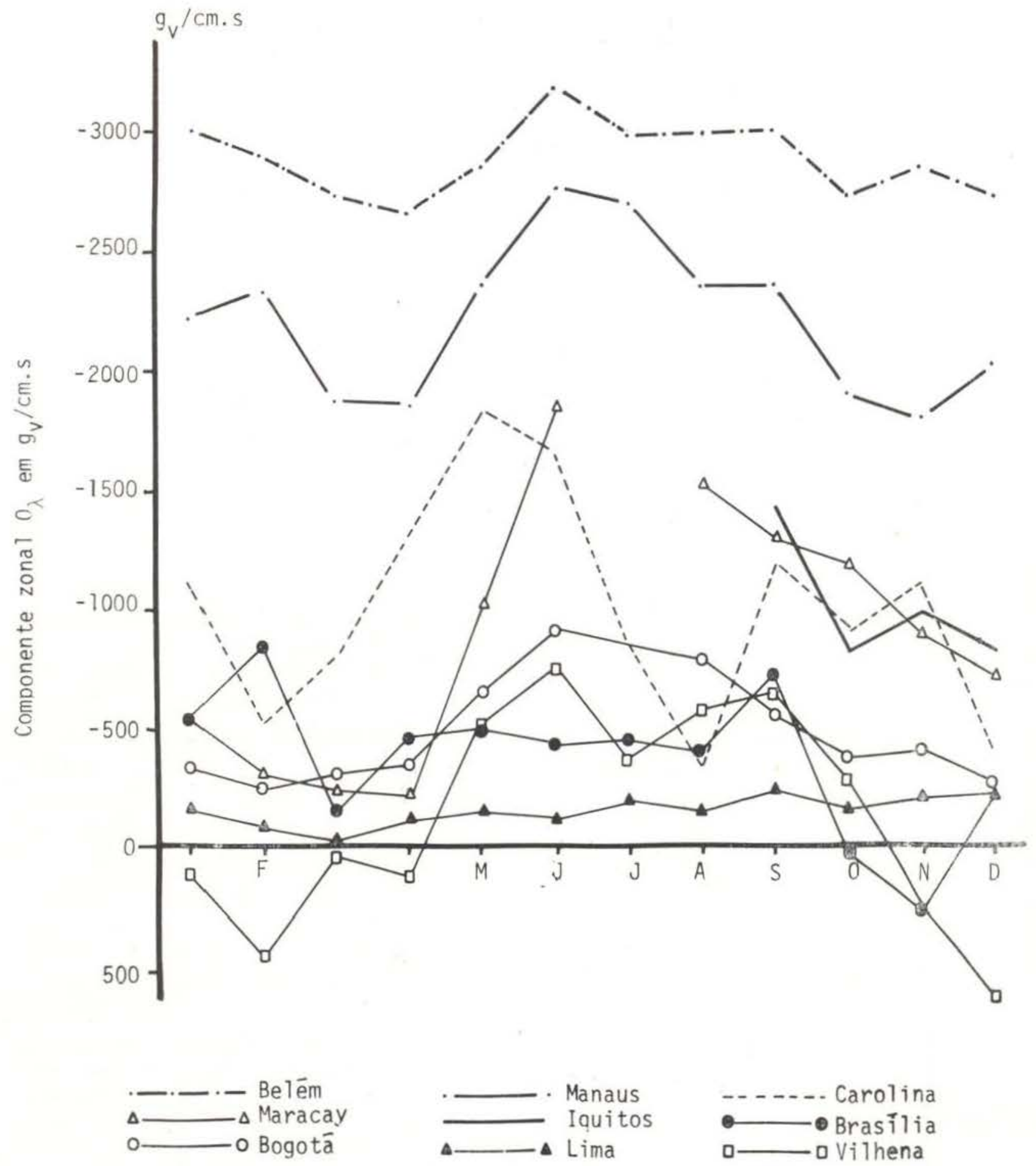

Fig. 2 - Distribuiçăo mensal da componente zonal $\mathrm{Q}_{\lambda}$ do fluxo de vapor, em $\mathrm{gv} / \mathrm{cm}$.s. Valores positivos indicam sentido de Oeste para Leste. 


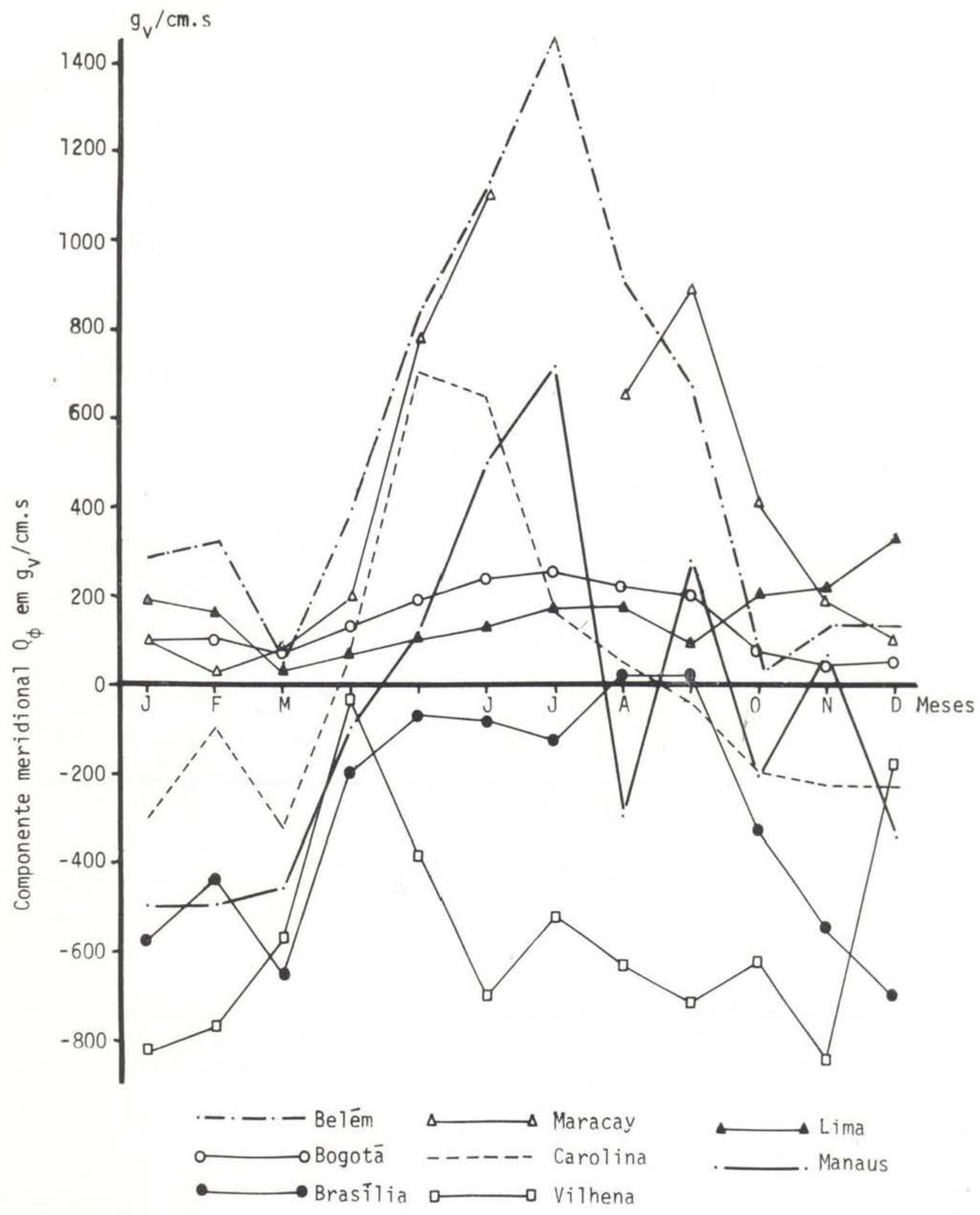

Fig. 3 - Distribuição mensal da componente meridional $\mathrm{Q} \phi$ do fluxo de vapor, em $\mathrm{gv} / \mathrm{cm} . \mathrm{s}$. Valores positivos indicam sentido de Sul para Norte. 
Os valores mensais das componentes zonal $\mathrm{O}_{\lambda}$ e meridional $\mathrm{Q} \phi$ do fluxo de vapor dágua atmosférico acham-se sumarizados na tabela 1.

DISTRIBUIÇĀo MENSAL DA COMPONENTE ZONAL DO FLUXO DE VAPOR, $Q \lambda$

A figura 2 construída com os valores médios de $Q_{\lambda}$ mostra a distribuição mensal da componente zonal do fluxo de vapor para algu. mas das localidades estudadas. Nota-se a predominância dos valores mais elevados em Beim e Manaus, constância do sentido leste oeste bem como o fato de estar a maioria das curvas em fase. Isto implica na caracterização de um comportamento uniforme do transporte de vapor no sentido leste-oeste na região.

\section{DISTRIBUIÇÃO MENSAL DA COMPONENTE} MÉRIDIONAL DO FLUXO DE VAPOR $ৎ \Phi$

Esta distribuição acha-se representada na figura 3. Com exceção de Vilhena, Brasília, Carolina e Manaus as demais apresentaram $Q \phi$ $\mathrm{ccm}$ sentido de sul para norte durante todo o ano, com valores maiores no inverno do he misfério sul.

\section{Distribuiçã̃ Do FLUXo TOTAL $\vec{Q}$ SOBRE A BACIA AMAZÔNICA}

A análise das figuras 4 a 7 evidencia que praticamente todo o ano é caracterizado por uma entrada maciça de vapor dágua na bacia. oriundo do Oceano Atlântico. Para o interior da bacia o fluxo vai diminuindo gradativamente, devido a diminuição da velocidade do vento pois há um aumento dos valores da umidade especifica à medida que maior penetração se verifica. Além disso o obstáculo natural que é a cordilheira dos Andes funciona como um verdadeiro paredão no fim de um hipotético corredor de quase $4.000 \mathrm{~km}$ de extensão. Nas mesmas figuras encontram-se também os campos do armazenamento aéreo do vapor dágua, estimado pela água precipitável $\mathrm{Wp}$.

$\mathrm{Da}$ análise das figuras os seguintes fatos sảo evidenciados :

- o papeel do oceano Atlântico com fonte

- constante de vapor dágua para a região;

- a regiăo Amazônica, por sua vez, exportando parte do vapor para a região do Chaco paraguaio e Brasil Central, prin-

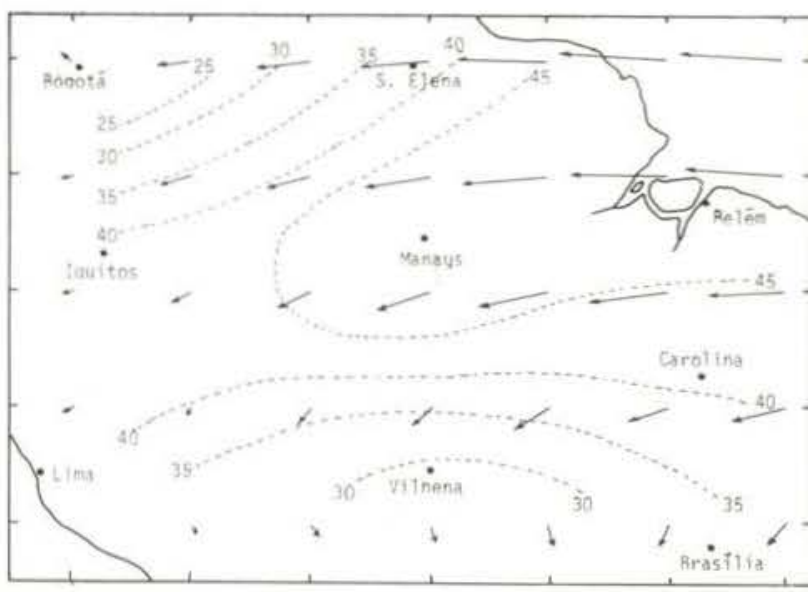

Fig. $4-$ Valores do campo vetorial $\overrightarrow{\mathrm{Q}}=\overrightarrow{\mathrm{Q}} \lambda \vec{\lambda}+\overrightarrow{\mathrm{Q}_{\phi}}$. Média do período $1972 / 1975$, obtida para as quadrículas de $5^{\circ}$ de latitude por $5^{\circ}$ de longitude. Valores para março. Linhas tracejadas: água precipitável, em $\mathrm{mm}$. $1 \mathrm{~cm}=2000 \mathrm{gv} / \mathrm{cm} . \mathrm{s}$

cipalmente nos meses de março e de zembro, sendo pequena esta contribuição em junho e setembro:

- apesar dos valores elevados do fluxo $\rightarrow$

$\overrightarrow{\mathrm{Q}}$ próximo a Belém, a existência de um período de seca bem definido na metade da illha de Marajó sugere a realização de estudos mais profundos e detaIhados das características do armaze mentc de água e circulação local.

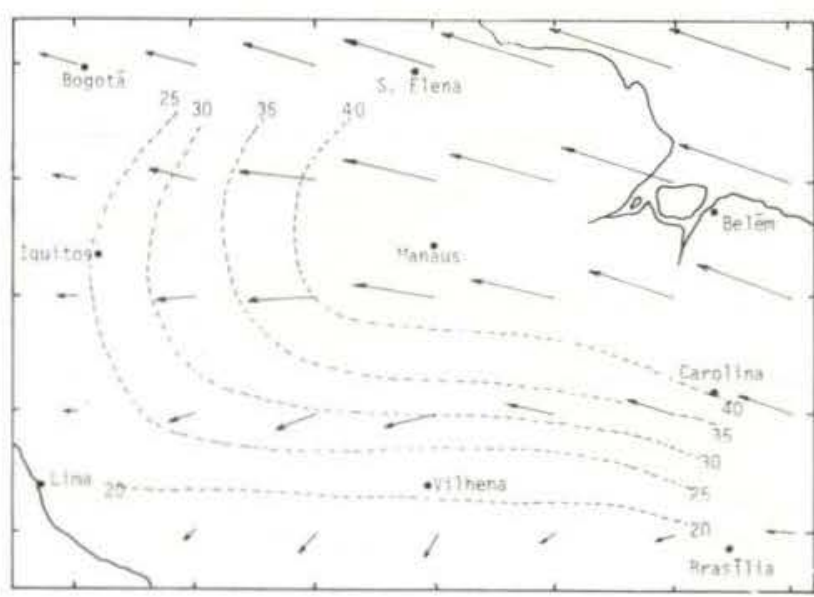

Fig. $5-$ Valores do campo vetorial $\vec{Q}=\overrightarrow{Q_{\lambda}}+\overrightarrow{Q_{\phi}}$. Média do período $1972 / 1975$, obtida para as quadrículas de $5^{\circ}$ de latitude por $5^{\circ}$ de longitude. Valores para junho. Linhas tracejadas: água precipitável, em $\mathrm{mm}$. $1 \mathrm{~cm}=2000 \mathrm{gv} / \mathrm{cm} . \mathrm{s}$ 


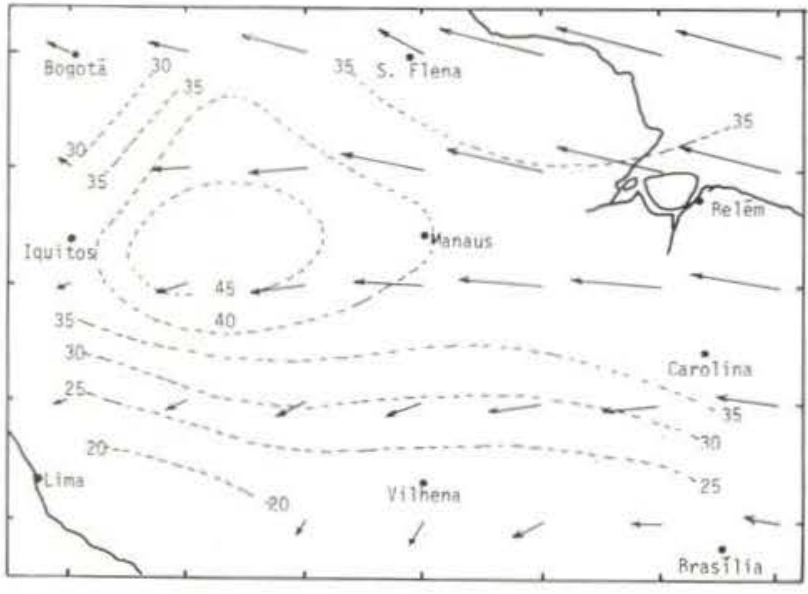

Fig. 6 - Valores do campo vetorial $\overrightarrow{\mathrm{O}}=\overrightarrow{\mathrm{O}_{\lambda}}+\overrightarrow{\mathrm{O}_{\phi}}$. Média do periodo $1972 / 1975$, obtida para as quadrículas de $5^{\circ}$ de latitude por $5^{\wedge}$ de longitude. Valores para setembro. Linhas tracejadas : água precipitável, em $\mathrm{mm}$. $1 \mathrm{~cm}=2000 \mathrm{gv} / \mathrm{cm} \cdot \mathrm{s}$

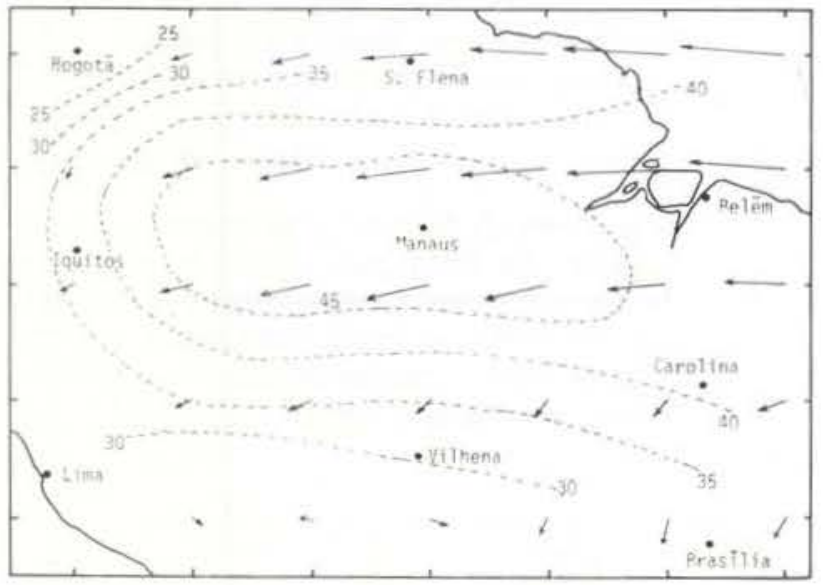

Fig. $7-$ Valores do campo vetorial $\overrightarrow{0}=\overrightarrow{Q_{\lambda}}+\overrightarrow{Q_{\phi}}$. Média do período $1972 / 1975$, obtida para as quadrículas de $5^{\circ}$ de latitude por $5^{\circ}$ de longitude. Valores para dezembro. Linhas tracejadas : água precipitável, em $\mathrm{mm}$. $1 \mathrm{~cm}=2000 \mathrm{gv} / \mathrm{cm} \cdot \mathrm{s}$

VARIAÇÃO MENSAL DA DISTRIBUIÇÃo VERTICAL JA COMPONENTE ZONAL DE VAPOR DÁGUA, $Q \lambda$

Nas figuras 8 a 11 acham-se representadas as variaçōes mensais e por altitude da componente zonal $\alpha \lambda$ para as principais estações utilizadas neste estudo.

As figuras 8 e 9 analisadas em conjunto mostram a predominância do sentido este-oes- te. na faixa Belém-Manaus. Os maiores vaiores acham-se em torno do nivel de $700 \mathrm{mb}$ o que corresponde a $2.400 \mathrm{~m}$ de altitude, aproximadamente. Desde a superfície até este nivel nota-se que o comportamento da variaçäo da componente $\mathrm{Q} \lambda$ é diferente daquele verificado entre os 750 e $500 \mathrm{mb}$.

A análise do trecho Brasilia-Vilhena, feita com auxilio das figuras 10 e 11 mostra uma concordância entre os perfis no período abrilsetembro mas de outubro a março Vilhena apresenta uma inversão no sentido do fluxo em todos os níveis. Naquele período, quando o domínio da ação do anticiclone permanente do Atlântico sul começa a se manifestar sobre o continente, há um enfraquecimento do centro de baixas pressões do Brasil Central e Chaco paraguaio e, em consequeência, impõe uma componente de Este. No periodo primavera-verão, o anticiclone desloca-se mais para o oceano dancio lugar a migração, em direção a leste do sistema de baixas pressões do Brasil Central aparecendo, então, uma componente oeste do vento principalmente sobre Vilhena. Este fato também acha-se evidenciado nas cartas de representação espacial do fluxo total (Figuras 4 a 7).

VARIAÇÃO MENSAL DA DISTRIBUIÇÃO VERTICAL DA COMPONENTE MERIDIONAL DO FLUXO DE VAPOR DÁGUA, $Q \Phi$

Em Belém, figura 12, o sentido norte-sul fez-se sentir somente na camada $1000 / 900 \mathrm{mb}$ exceto no período do inverno; a partir dos $900 \mathrm{mb}$ toda penetração longitudinal de vapor na bacia se processou do sul para norte. A costa nordeste da América do Sul teve uma adição constante de vapor dágua oceânico, sem alterar a característica quase latitudinal do fluxo total $\vec{Q}$, conforme pode ser verificado pelas figuras 4 a 7 .

A análise do trecho Belém-Carolina, figuras 12 e 13, evidenciou que, no verão, o deslocamento para leste do centro de altas pressōes do Atlântico sul altera notadamente o vento naquele trecho tanto em direção como em velocidade, permanecendo inaiterado os valores da umidade específica. 


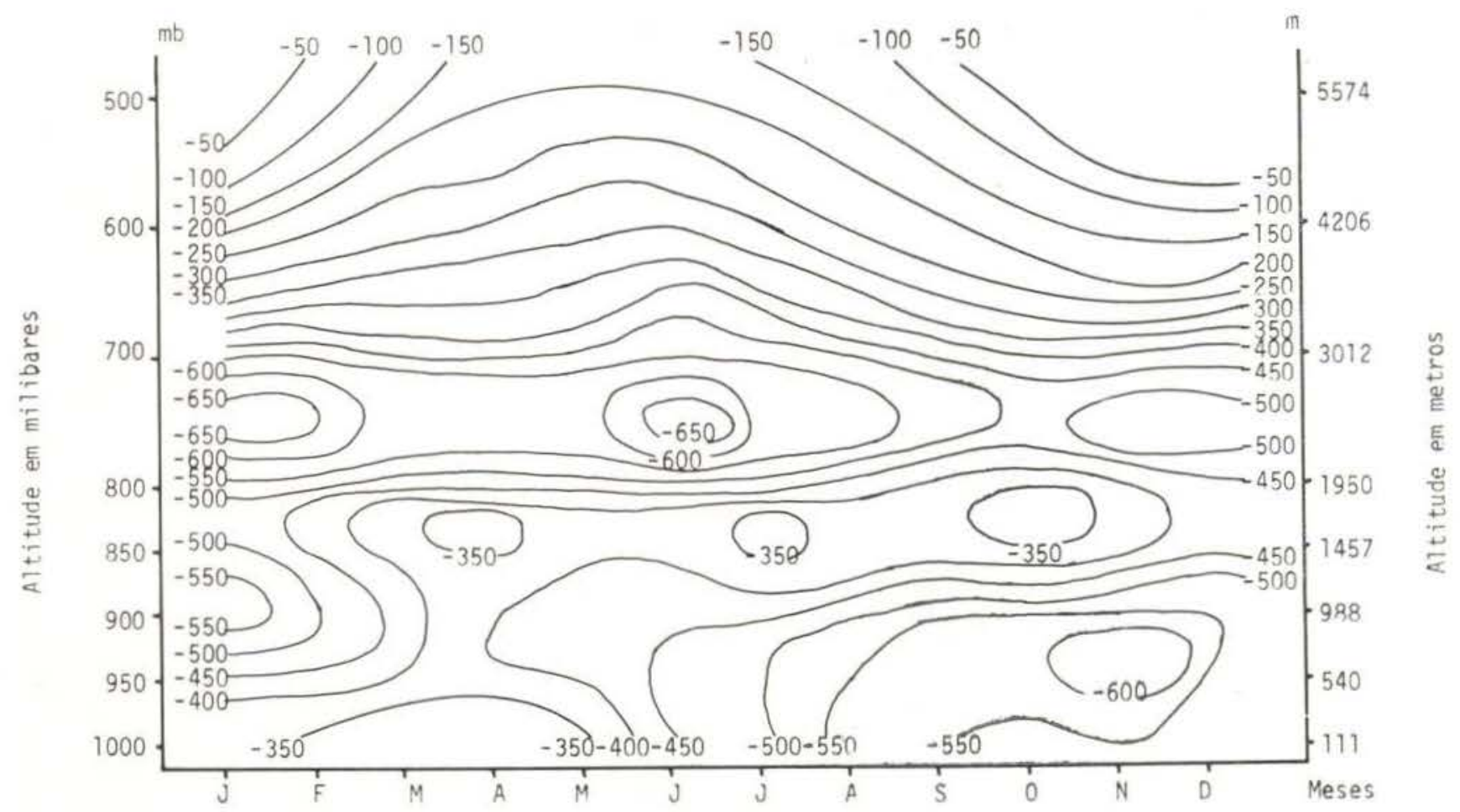

Fig. 8 - Variação mensal da distribuiçăo vertical da componente zonal $\mathrm{O} \lambda$, em $\mathrm{gv} / \mathrm{cm}$.s, em Belém. Média do periodo 1972/1975. Os valores negativos indicam sentido de Leste para Oeste.

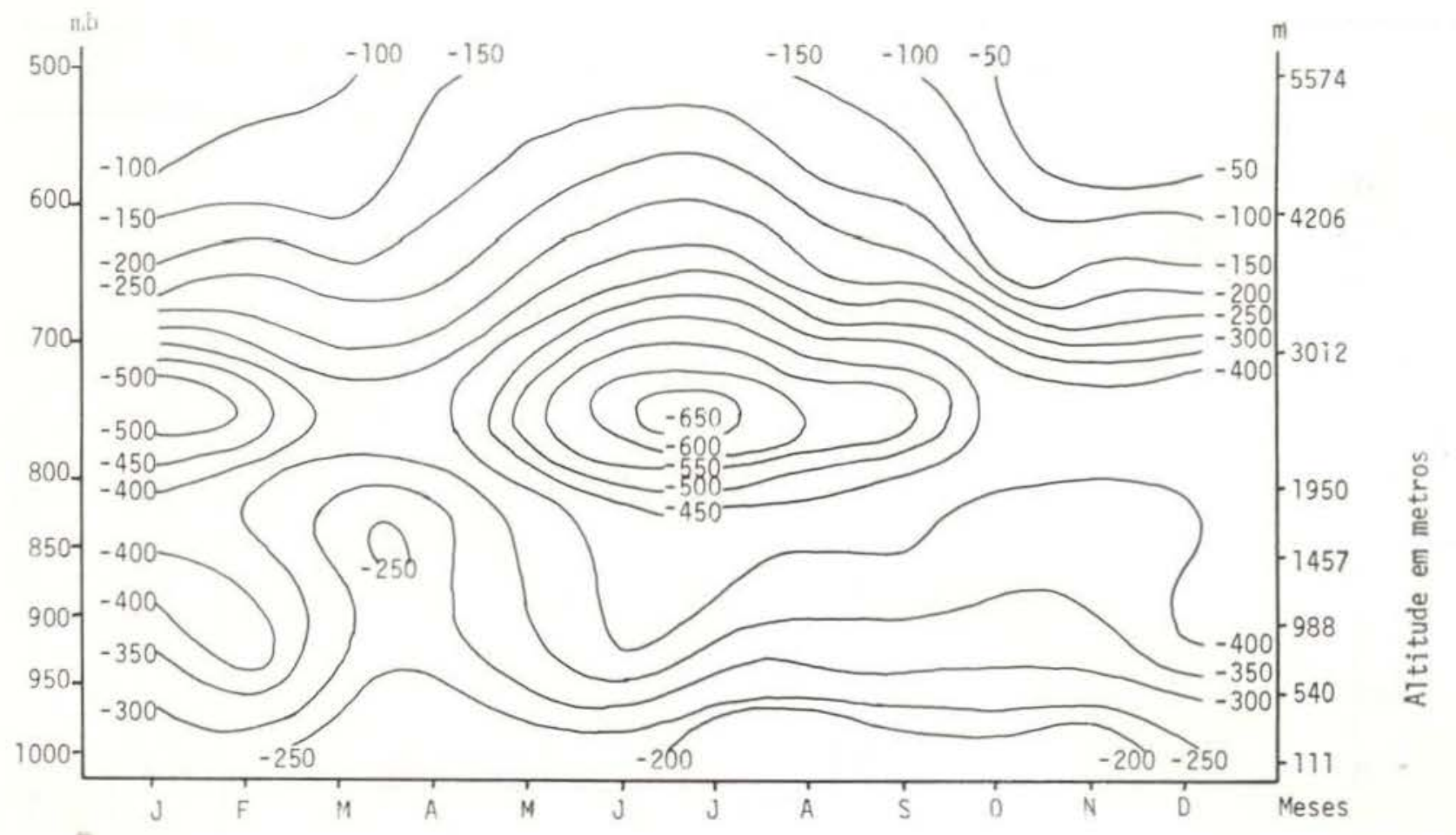

Fig. 9 - Variação mensal da distribuição vertical da componente zonal $\mathrm{Q} \lambda$, em $\mathrm{gv} / \mathrm{cm} . \mathrm{s}$, em Manaus. Média do período 1972/1975. Os valores negativos indicam sentido de Leste para Oeste. 
OS PERFIS DAS COMPONENTES DO FLUXO DE VAPOR DÁGUA AO LONGO DA BACIA AMAZÔNICA

As operações de radiossondagem de Iquitos, Peru. iniciaram-se em setembro de 1975 , aumentando o número de informações aerológicas disponiveis, principalmente na América do Sul, cuja baixa densidade da rede não tem permitido um maior detalhamento da circula ção geral da atmosfera nas regiōes tropical $\Theta$ equatorial. Afortunadamente lquitos, situada na bacia Amazônica constitui-se, juntamente com Manaus e Belém, importantes pontos que

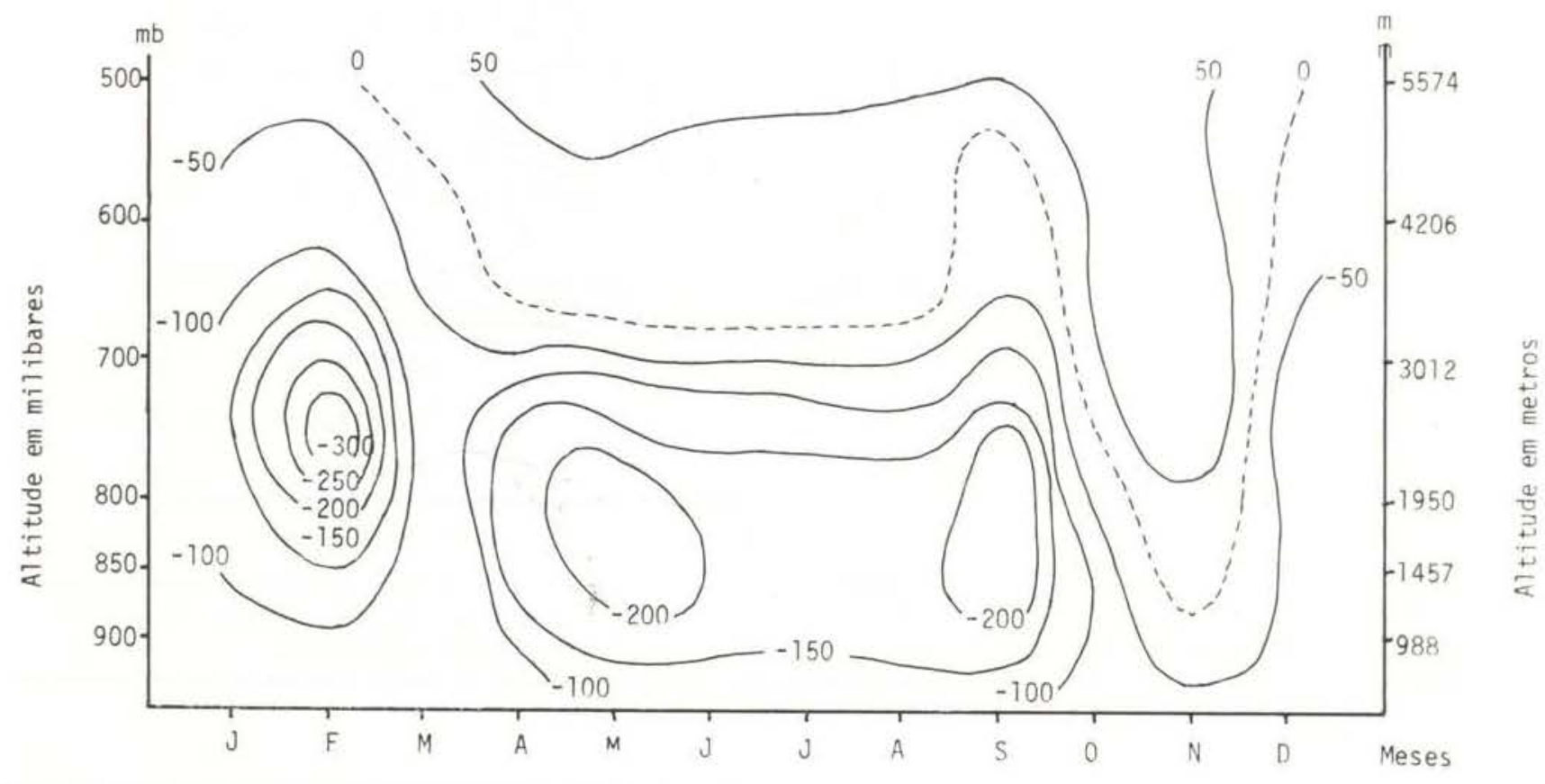

Fig. 10 - Variação mensal da distribuiçăo vertical da componente zonal $\mathrm{Q} \lambda$, em $\mathrm{gv} / \mathrm{cm} . \mathrm{s}$, em Brasília, Média do período 1972/1975. Os valores negativos indicam sentido de Leste para Oeste.

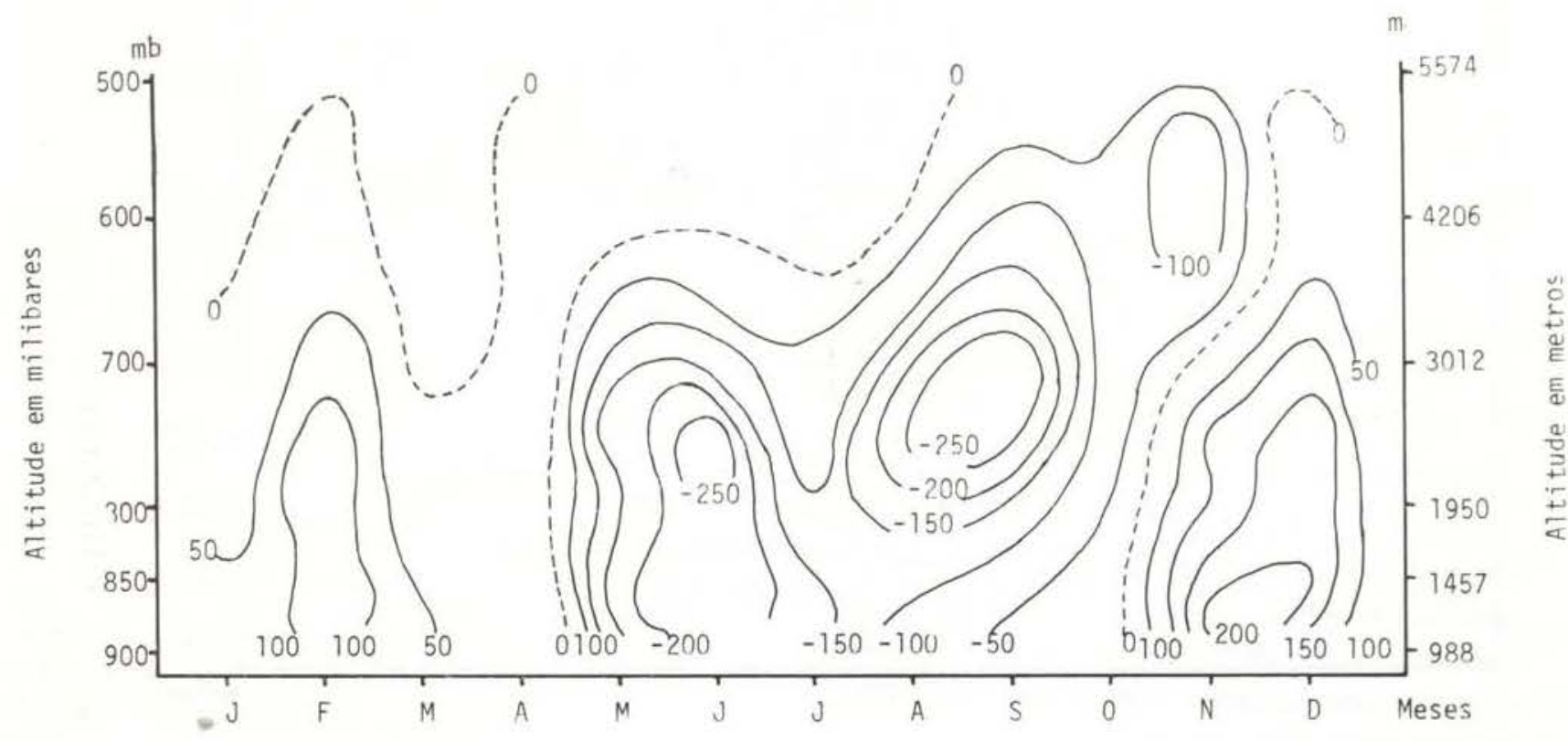

Fig. 11 - Variação mensal da distribuição vertical da componente zonal $Q \lambda, \mathrm{cm} \mathrm{gv} / \mathrm{cm} . \mathrm{s}$, em Vilhena. Média do periodo 1972/1973. Os valores negativos indicam sentido de Leste para Oeste. 


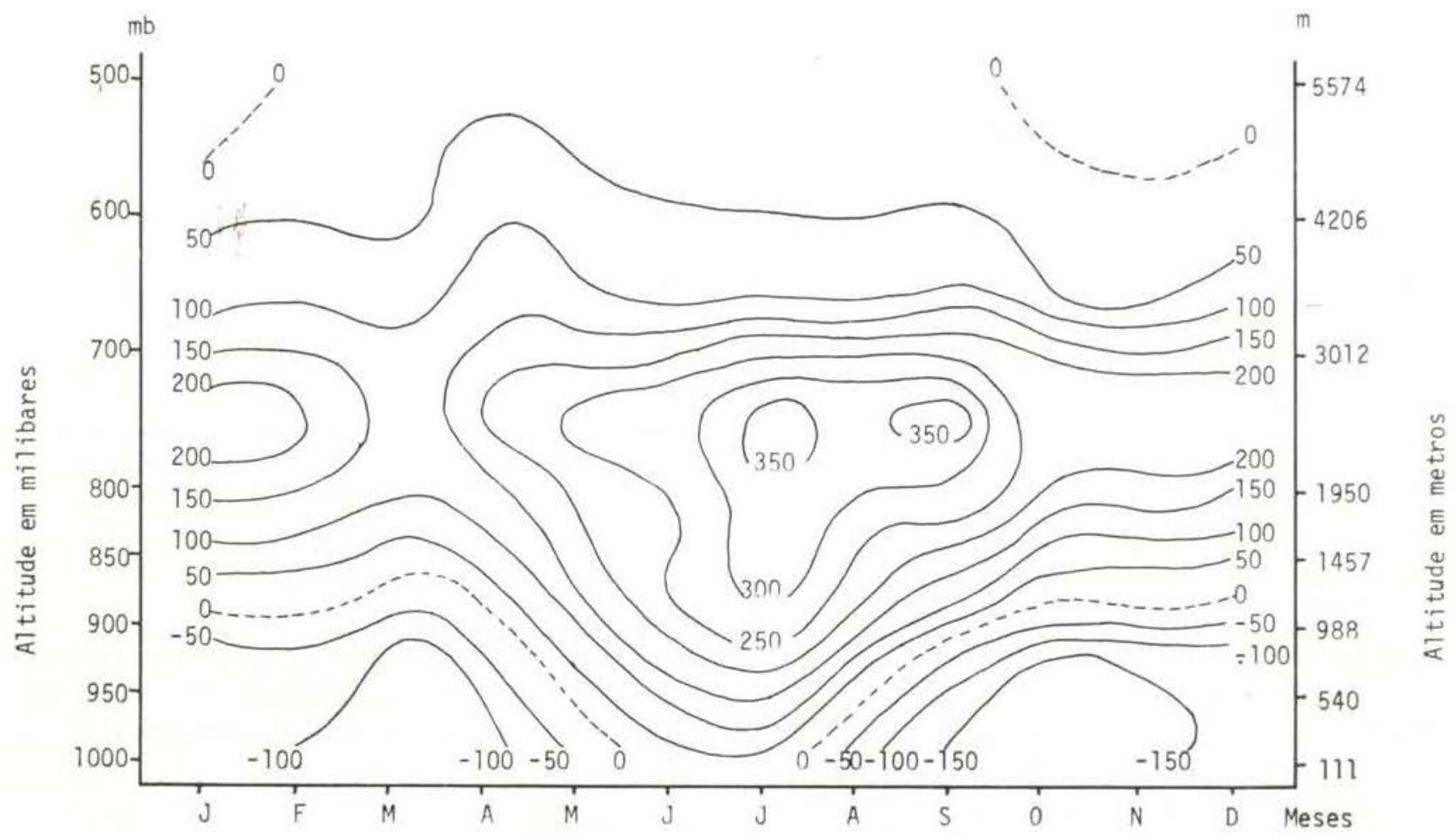

Fig. 12 - Variação mensal da distribuição vertical da componente meridional $\mathrm{Q} \phi$, em $\mathrm{gv} / \mathrm{cm}$.s, em Belém. Média do período 1972/1975. Os valores negativos indicam sentido de Norte para Sul

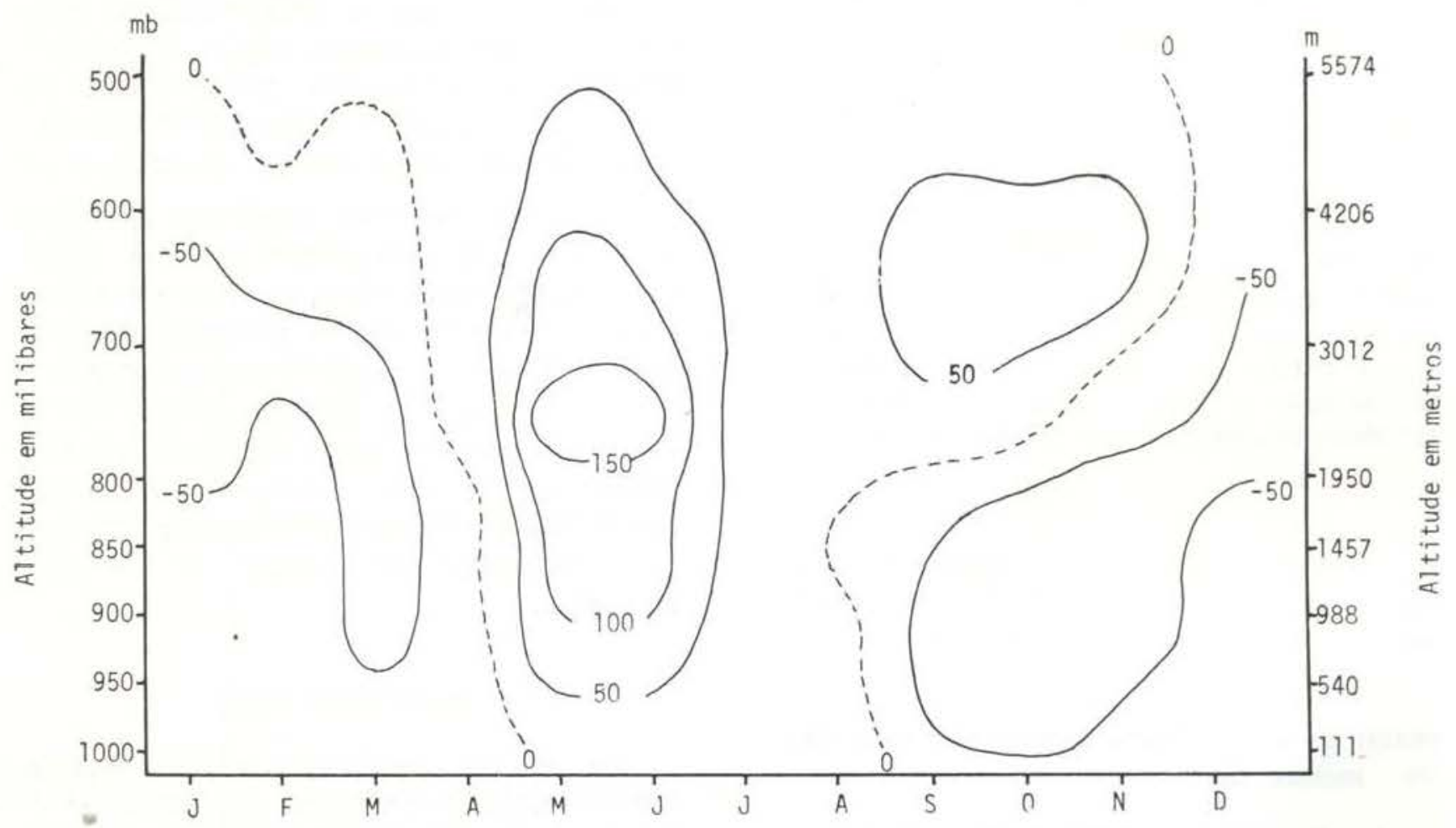

Fig. 13 - Variação mensal da distribuição vertical da componente meridional $Q \phi$. em gv/cm.s, em Carolina. Média do periodo $1972 / 1974$. Os valores negativos indicam sentido de Norte para Sul. 
p€rmitirão uma melhor representação e entendimento da distribuição dos transportes de vapor dágua na região. As figuras 14 e 15 foram construídas somente com os dados do mês de setembro de 1975 não devendo serem consideradas como representativas de uma situação climatológica para a área.

Nas representações mensais, não apresentadas neste trabalho, verificou-se uma repetição anual do mesmo modelo de perfil, sugerindo que as figuras acima não se distanciaram muito do padrão.

PERFIL DA COMPONENTE ZONAL DO FLUXO, $0 \lambda$. FIGURA 14

Houve uma diminuição dos valores do fluxo zonal, com gradiente bem acentuado à medida que maior penetração para o interior é verificada. Acima dos $650 \mathrm{mb}(\simeq 3.500 \mathrm{~m})$ a componente zonal do fluxo inverte o senti-

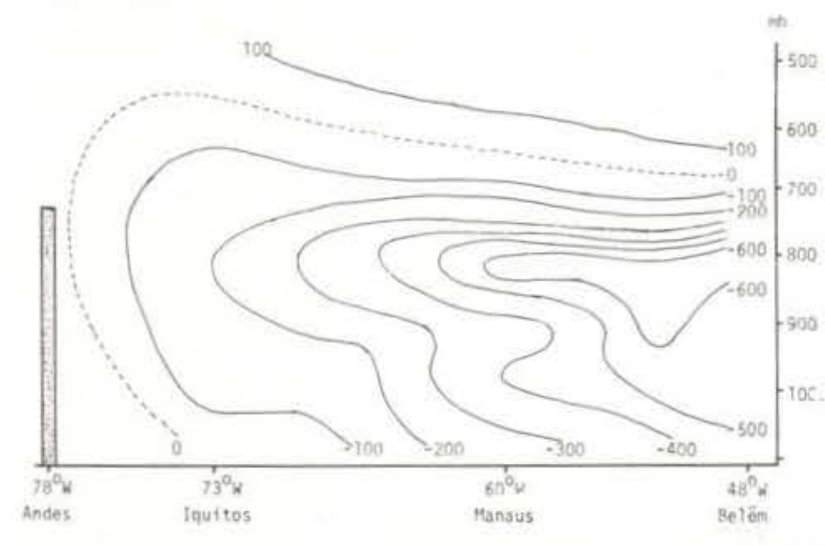

Fig. 14 - Perfil longitudinal da componente zonal oio fluxo de vapor $Q \lambda$, para setembro/ 1975, em gvcm.s. Valores negativos indicam sentido Leste-Oeste.

do, passando a ser de oeste para leste. Nos niveis mais baixos e entre Iquitos e a CordiIheira dos Andes o valor tende a se anular.

PERFIL DA COMPONENTE MERIDIONAL DO FLUXO, Od, FIGURA 15

Verificoū-se a existência de uma componente, da magnitude relativamente grande, no sentido da Amazônia para o interior do con-

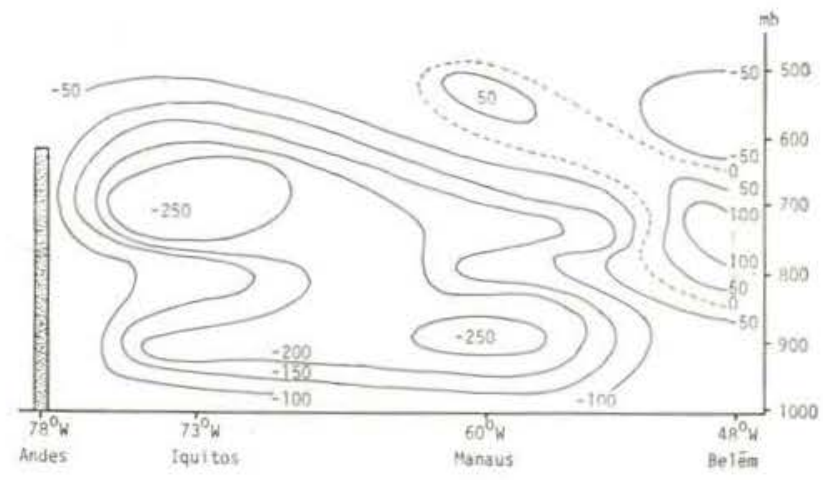

Fig. 15 - Perfil longitudinal da componente meridional do fluxo de vapor $\mathrm{Q} \phi$, para setembro/1975, em gv/cm.s. Valores negativos indicam sentido Norte-Sul.

tinente significando que, pelo menos no mês analisado há uma exportaçăo de vapor da Amazônia para o interior do continente.

\section{CONCLusões}

Os resultados aqui apresentados resumense aos obtidos para um período relativamente curto (1972-1975), mas que, de um modo gerál, dão uma idéia do comportamento dinâmico do vapor dágua na região Amazônica. Estudos mais detalhados, principalmente na parte mais oriental da bacia deverão ser feitos abrangendo maior número de anos.

As conclusões mais genéricas são aquelas relativas à constatação de uma adição constante de vapor dágua de origem oceânica e com uma componente predominantemente zonal. Entretanto, segundo pode-se depreender do trabalho de Peixoto (1972) não é descartada a possibilidade de alguma penetração de vapor dágua oriundo do oceano Pacífico. possibilidade esta que deverá ser estudada para um melhor entendimento da hidroiogia da região.

\section{AGRADECIMENTOS}

Os autores agradecem a FINEP, CNPq, FAPESP, CNEN e Secretaria de Tecnologia do Estado de São Paulo pelos recursos concedidos; à SUDENE, Ministério da Aeronáutica (Divisão de Meteorologia/DEC e DEC/CTA) e 
Força Aérea Venezuelana pela cessão dos dados meteorológicos de altitude necessários a esta pesquisa.

\section{SUMMARY}

The zonal $Q_{\lambda}$ and meridional $\mathrm{O}_{\phi}$ water vapor flux components, from 1972 to 1975 period, using 9,348 radiossonde data are presented; there is a zonal water vapor flux evidence over the Amazonas basin. The mean $\mathrm{O}_{\lambda}$ values over Belém and Manaus were 2,895 and $2,203 \mathrm{~g} / \mathrm{cm}$.s, respectively, in a $1000 / 500 \mathrm{mb}$ layer.

\section{BibLIOGRAFIA}

BARNES, A.C. JR.

1964 - Atmcspheric water vapor divergence: measurements and aplications. In: Humidity and moisture, N.Y., Reinh. Publ. Co. 2. pag. 513-527.

Benton, G.S.; Blackburn, R.T. \& Snead, V.O.

1950 - The role of the atmosphere in the hydrology cycle. Trans. Am. Geophys. Un. $31: 61-73$.

Benton, G.S. \& Estoque, M.A.

1954 - Water-vapor transfer over the North American Continent. J. Met. $11: 462-477$.

Bettencourt, M.C. \& FARIA, J.M.R.

1970 - The water vapor in the atmosphere and the hydrological cycle. Idöjárás Budapest, (5-6) : 307-312.

Hutchings, J.W.

1961 - Water-vapor transfer over Australian Continent. J. Met., $18: 615-634$.

MARQUES, J.

1976 - Contribuição ao estudo hidrológico da bacia Amazônica. Dissertaçāo MS, Escola Superior de Agricultura "Luiz de Queiroz", Universidade de São Paulo, 116 p.
Marques, J.; Santos, J.M, dos; Villa Nova, N.A. \& SALATT, E.

1977 - Precipitable water and water vapor flux between Belém and Manaus. Acta Amazonica, Manaus, $7(3): 355-362$.

Molion, L.C.B.

1975 - A climatonomic study of the energy and moisture fluxes of the Amazonas basin with considerations of deforestation effects. Ph.D Thesis, Univ. Wisconsin, Madison, $133 \mathrm{p}$.

Palmén, E. \& Söderman, D.

1966 - Computations of the evaporation from Baltic Sea from the flux of water vapor in the atmosphere. Geophysica, 8:261-279.

Peixoto, J.P.

1959 - O campo da divergência do transporte do vapor de água na atmosfera. In: Rev. Fac. Ciências de Lisboa, Separata 29, série B, Vol. 7, p. 25-56.

PEIXoto, J.P.

1972 - Pole - to pole water balance for the IGY from serological data. Nordic Hydrology, Copenhagen, $3: 22-43$.

RAsmusson, E.M.

1966 - Diurnal variation in the summer water vapor transport over North America. Water Res. Research, 2(3) : 469-477.

1967 - Atmospheric water-vapor transport and the water balance of North America. Month Wea. Rev., $95: 403-425$.

SPAR, J.A.

1953 - A suggested technique for quantitative precipitation foreasting. Month Weather Rev., $81: 217-221$

(Aceito para publicação em 28/09/79) 